\title{
Hybrid-Data-Driven Fuzzy Recognition of Operator Cognitive State in Human-Machine Cooperative Control System
}

\author{
Jian-Hua Zhang*, Jörg Raisch ${ }^{* *}$ \\ *Dept. of Automation, East China University of Science and Technology, Shanghai 200237, P.R. China \\ (Tel: ++86-21-64253808; e-mail: zhangjh@ecust.edu.cn) \\ **Control Systems Group, Technische Universität Berlin, D-10587 Berlin; Systems and Control Theory \\ Group, Max Planck Institute for Dynamics of Complex Technical Systems, D-39106 Magdeburg, \\ Germany
}

\begin{abstract}
In this paper, the Human Cognitive State (HCS) in the context of human and complex system interaction was classified by using the Fuzzy C-Means (FCM) algorithm. The most important features for the HCS pattern classification were extracted from a set of measured physiological variables based on the comparison of their respective rates of correct classification. The results have shown the usefulness of the FCM algorithm as well as the selected features for the challenging HCS classification problem.
\end{abstract}

Keywords: Man-machine systems; Cognitive science; Physiology; Fuzzy set theory; Pattern recognition; Classification

\section{INTRODUCTION}

In the safety-critical human-machine systems, the serious consequences of the decrement or breakdown of the human (operator) performance have drawn world-wide attention and concern, especially in the fields of transportation (e.g., railway, shipping, aeronautics and space) and process industry (e.g., chemical plants and nuclear power stations). A possible solution to this problem is to adjust the control strategy of such systems based on the analysis of the Human operator's Cognitive State (HCS) (Hockey, 2003). In order to enhance the overall performance of such systems, an adaptive adding system can be developed, based on the identification or recognition of the HCS, to either warn the operator or reduce the task demands on the operator during the period of excessively high Mental WorkLoad (MWL). In Nickel et al. (2005) and Nickel et al. (2006), it was shown that some psychophysiological markers, for instance EEG-based-TLI (Task Load Index), are quite sensitive to the level of the operator's mental stress or workload. Wilson (1999) adopted Artificial Neural Network (ANN) based approach to classification of operator functional (psychological or cognitive) state. Nonetheless, their approach can only provide the information regarding to which class a momentary operator state belongs to. Due to the black-box (and thus hard-to-interpret) nature of the ANN approach as well as the uncertain and fuzzy characteristics of the HCS classification problem, the ANN-based approach failed to provide a practically feasible solution to the difficult problem of recognizing the HCS in an accurate as well as transparent way. In this paper, based on a set of measured psychophysiological data (ECG, EEG, etc.), the Fuzzy C-Means (FCM) algorithm, originally proposed by Bezdek (1981), was employed to perform the HCS classification, which not only gives the cluster information but also the degrees of membership of a certain class.

\section{EXPERIMENTAL DATA}

\subsection{Data collection}

In this work, an automation-enhanced Cabin Air Management System (aCAMS) was used to simulate a highly complex and safety-critical multi-task process control environment. The aCAMS was originally developed for ESA (European Space Agency) for investigating the stressors of the astronauts in the highly separated and confined environment (Hockey et al, 1998). In aCAMS, the primary tasks of the human operator are to control (regulate) the five key controlled variables within the targeted ranges, and to handle in real time the programmed problems or faults (e.g., the operator is required to perform manual control tasks to maintain the normal operation of the whole system once an automatic controller or control subsystem malfunctions).

Prior to the formal data acquisition experiments, each subject had undergone training of manual process control tasks for more than $10 \mathrm{hrs}$ to get familiar to the experimental environment and control tasks. A total of 10 subjects (with subject-code A, C, D, E, F, G, H, J, K, and L, respectively) finally participated in the formal experiments, each carrying out 2 sessions of experiment. Each experimental session consisted of 9 task-load conditions, each condition lasting for $15 \mathrm{~min}$, hence each session lasted for $135 \min (=15 * 9)$. The experimental session was designed to emulate different task environments and task-loads according to the so-called cyclical loading scheme and consists of 9 different task-load conditions, under each of which there were a certain number of controlled variables demanding manual control by the operator (i.e., C1-C9 corresponds to the cyclic number of 1, 2, $3,4,5,4,3,2$, 1, respectively). Prior to each task-load condition, the subject was asked to fill in health status questionnaire and complete subjective ratings of such variables as the level of efforts, anxiety and fatigue in a 
manner of self-report on the relevant scales (in the range of 0 to 100 points with 0 and 100 representing the two extremes of the lowest and highest level, respectively).

In the whole session, the electroencephalogram (EEG), electrocardiograph (ECG) and operator performance data were recorded continuously. The EEG data was collected at a sampling rate of $2048 \mathrm{~Hz}$ according to the international 10-20 electrode placement system (Jasper, 1958). For each EEG electrode (or measurement channel), three band powers, namely $\theta(4-8 \mathrm{~Hz}), \alpha(8-13 \mathrm{~Hz})$, and $\beta(13-22 \mathrm{~Hz})$, were calculated. The data preprocessing was performed on the Biosemi ${ }^{\circledR}$ system. The preprocessed and down-sampled heart rate (HR) and EEG data was recorded every second.

The instantaneous values of the five key controlled variables (i.e., multiple process outputs) were also measured, which can be used to derive the primary-task performance of the human operator who shared the control tasks with the machine in the whole human-machine system.

\subsection{Data preprocessing}

Considering the time period of subjective ratings, the first and last 30-sec measurement data were discarded, hence each task-load condition contains 14-min data and we had the usable time-series data of length $14 \times 9=126 \mathrm{~min}$ for each experimental session.

Based on the preprocessed HR data, we define the following heart rate variability (HRV) index, $\mathrm{HRV}_{2}$, as the ratio of the standard deviation and the mean of the HR data within a sampling interval (i.e., $1 \mathrm{~min}$ ), which can be computed by (Wilson, 1999):

$$
H R V_{2}=\frac{\sigma_{H R}}{\mu_{H R}}
$$

where $\sigma_{H R}$ and $\mu_{H R}$ stand for the s.d. and mean value of the HR time-series data within the time interval considered.

Furthermore, the frequency spectral analysis of the HRV time-series data was performed to compute the ratio of lowerand higher-frequency band powers, i.e., LF/HF (Zhang et al, 2008), where the LF and HF bands are defined as $0.03-0.15 \mathrm{~Hz}$ and $0.18-0.4 \mathrm{~Hz}$, respectively.

For EEG data, the following two TLI indices (Wilson, 1999) were calculated by:

$$
\left\{\begin{array}{l}
T L I_{1}=\frac{P_{\theta, F z}}{P_{\alpha, P z}} \\
T L I_{2}=\frac{P_{\theta, A F z}}{P_{\alpha, C P z P z}}
\end{array}\right.
$$

where $\mathrm{P}_{\theta}$ and $\mathrm{P}_{\alpha}$ stand for the theta- and alpha-band power, respectively, with the division of the specific frequency bands at five different electrode sites (in standard 10-20 EEG electrode placement system) defined as: $(\mathrm{Fz}, \theta): 6-7 \mathrm{~Hz}$; $(\mathrm{AFz}$, ө): 5-7 Hz; $(\mathrm{Pz}, \alpha): 10-12 \mathrm{~Hz} ;(\mathrm{CPz}, \alpha): 8-10.5 \mathrm{~Hz} ;(\mathrm{POz}, \alpha)$ :

\section{$10-13.5 \mathrm{~Hz}$.}

All data was normalized by:

$$
\left\{\begin{array}{l}
z^{\prime}=\frac{z-z_{\min }}{L_{z}} \\
L_{z}=z_{\max }-z_{\min }
\end{array}\right.
$$

where $z^{\prime} \in[0,1]$ is the normalized version of the original data $z$, and $z_{\max }$ and $z_{\min }$ are the maximal and minimal value of the data set.

\section{HCS CLASSIFICATION BASED ON FCM ALGORITHM}

\subsection{Fundamental ideas}

The HCS is referred to as the cognitive (mental or psychological) state of the human operator, which has many different correlates including possibly the physiological state, psychological state, the task demand, task environment, and other external factors. All those factors determine the performance of the operator when dealing with the current tasks under the current state and environment. In this work, the HCS evaluation is quantified and indexed by the task (work) performance of the operator. A variety of pattern recognition methods have been proposed. This paper adopted fuzzy c-means algorithm to perform the HCS classification based on the following two main reasons:

1) The intrinsic nature and characteristics of the HCS pattern recognition problem require the use of fuzzy logic based approach with more flexible (or softer) class membership. In practical estimation of the HCS based on the psycophysiological measures (response), a linguistic variable, such as 'excellent', 'average', 'worse', may be usually used to describe or differentiate the levels of the HCS. Even in some cases, only a 'hard-threshold-like' redline of the operator's task performance needs to be determined to infer the optimal allocation of the tasks to the operator, so as to avoid the operational accidents caused by the deterioration of the operator state. Therefore, the fuzzy logic based HCS pattern classification problem is mainly considered.

2) The FCM algorithm has moderate computational overhead Furthermore, in the FCM, all variables are continuous and differentiable and thus gradient-based technique can be used to find a viable and more efficient search direction, which avoids the blind search in the whole feature space and thus the combinatorial explosion issue related to other traditional non-fuzzy approaches.

\subsection{Basics of FCM algorithm}

The fuzzy C-Means (FCM) algorithm is concerned with partition-based clustering and unsupervised learning. The clustering is performed completely based on the inter-relationship of data itself without the need of explicit information about the target clusters. The basic idea of the FCM algorithm is to maximize the similarity of the objects 
which are partitioned to a certain cluster, whereas to minimize the similarity between different clusters.

The FCM algorithm improved the normal c-means clustering algorithm in terms of the data partition approach with the latter adopting ' $h a r d$ ' partition, whereas the former 'soft' partition by means of fuzzy logic. In the normal c-means algorithm, certain data point either belongs to or does not belong to a cluster. In contrast, in the FCM algorithm each data point belong to every cluster to different degrees (referred to as the membership degree represented by a value between 0 and 1) with the sum of all membership degrees equal to 1 . The closer to 1 the membership degree, the more significant the membership of the data to this cluster is.

The specific computational procedure of the FCM algorithm is as follows:

Step 1: Given the data set $X=\left[\mathbf{x}_{1}, \mathbf{x}_{2}, \cdots, \mathbf{x}_{n}\right]$ with $\mathbf{x}_{k} \in R^{p \times 1}, k=1,2, \cdots, n$, preset the number of clusters $c \in\{2,3, \cdots, n-1\}$ and weight $m \in(1, \infty)$, and initialize the membership degree (MD) matrix $U^{(0)} \in M_{f c}$ with $M_{f c}$ the fuzzy c-partition of X.

Step 2: at the $l$-th $(l=0,1, \cdots)$ iteration, compute the $c$ cluster centers (vectors) by

$$
\mathbf{v}_{i}^{(l)}=\frac{\sum_{k=1}^{n}\left(u_{i k}^{(l)}\right)^{m} \mathbf{x}_{k}}{\sum_{k=1}^{n}\left(u_{i k}^{(l)}\right)^{m}}, 1 \leq i \leq c
$$

Step 3: Update $U^{(l)}=\left[u_{i k}^{(l)}\right]_{c \times n}$ to $U^{(l+1)}=\left[u_{i k}^{(l+1)}\right]_{c \times n}$ by:

$$
\begin{aligned}
& u_{i k}^{(l+1)}=\frac{1}{\left(\left\|\mathbf{x}_{k}-\mathbf{v}^{(l)}\right\|\right)^{\frac{2}{m-1}}}, 1 \leq i \leq c ; 1 \leq k \leq n \\
& \sum_{j=1}^{c}\left(\frac{\left\|\mathbf{x}_{k}-\mathbf{v}_{i}^{(l)}\right\|}{\left\|\mathbf{x}_{k}-\mathbf{v}_{j}^{(l)}\right\|}\right)^{\overline{m-1}}
\end{aligned}
$$

Step 4: If $\left\|U^{(l+1)}-U^{(l)}\right\|<\varepsilon$ (here $\varepsilon$ is a small constant) is satisfied or the preset number of iterations are completed, the algorithm stops and outputs the clustering results, otherwise let $l+1 \rightarrow l$, and then back to the Step2 to continue the computational loop.

The above four computational steps constitute the flowchart of FCM algorithm, which is shown in Fig. 1.

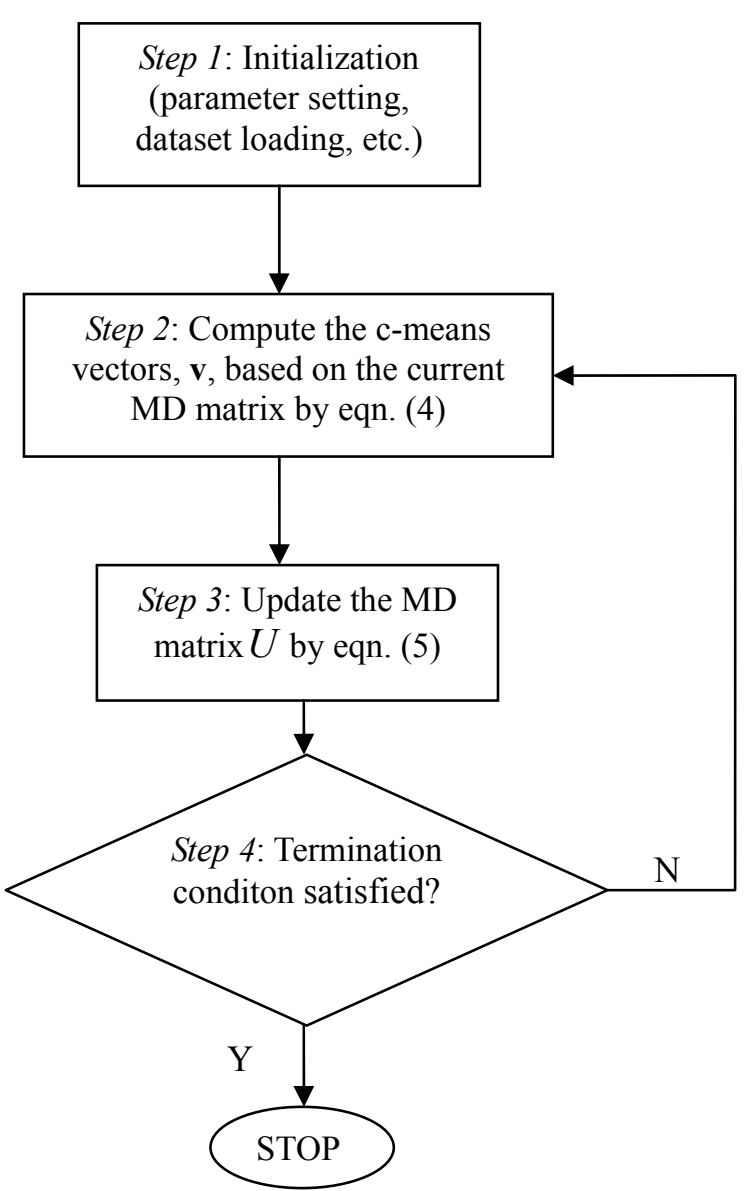

Fig. 1. The flowchart of the FCM algorithm.

\subsection{Results and some discussion}

The HCS classification by using the FCM algorithm gives us not only the class of each momentary state, but also the degree of the class membership as well as the center of each cluster. The class with maximal membership would be selected as the final class to which certain data point belongs. In our work, $c=3$, i.e., the HCS was classified into three classes with label of Risky, Average and Good, respectively. Based on the measured data, a total of 5 features were derived, including $\mathrm{HRV}_{2}, \mathrm{LF} / \mathrm{HF}, \mathrm{TLI}_{1}, \mathrm{TLI}_{2}$, and $\mathrm{HR}$ (the average value of heart rate at each minute). In this case, $p=5$, which implies that the dataset consists of the data points in 5-D feature space. In what follows, the HCS classification results for three subject $(\mathrm{D}, \mathrm{K}$ and $\mathrm{L}$ ) among a total of 10 subjects will be demonstrated. The five HCS features are shown in Fig. 2, 4, 6, 8, 10, 12a), respectively, while the corresponding FCM-based data clustering results are shown in the panel b) of the same figures by small squares.

To evaluate the effectiveness of the FCM algorithm for the HCS classification, a supplementary variable NOV (Number Of Variables under manual control) is considered as a reference. In our experimental design, we varied the NOV to realize a cyclical loading scheme (i.e., the NOV was stepwise increased until a threshold, then gradually reduced in a cyclic manner, refer to the same solid stepwise line in Fig. 2b, etc.). In this way the HCS variations with different task- and mental-load conditions can be thoroughly investigated. 
It can be seen from Fig. 2, 4, 6, 8, 10, 12b) that the HCS gradually deteriorates with the elevation of task-load during the first half period of the experimental session, whereas it gradually recovers with the reduction of the task-load during the latter phase of the session. The actual variations in the HCS level are well captured by the FCM-based classification results. The HCS classification results of the other seven subjects also reveal its real variations, thus the validness of the selected five features used for the HCS classification problem is basically substantiated.

In what follows, the difference between the cluster centers is used to further examine the sensitiveness of each candidate feature-set to the HCS classification. In other words, certain relatively insensitive features can be eliminated based on the information with regard to the cluster centers, so as to reduce the computational complexity of the FCM algorithm. By measuring the inter-cluster difference, some features that are relatively insensitive to the HCS variations can be removed. In this work, the feature(s), resulting in too close cluster centers measured by their Euclidean distance in (6), would be eliminated:

$$
\left\|\mathbf{v}_{i}-\mathbf{v}_{j}\right\| \leq \delta, i \neq j^{\prime} ; i, j=1,2, \cdots, c
$$

where $\delta$ is a small positive threshold that controlled the number of resulting clusters. Here we take its value to be 0.1 .

The reduced feature space after feature elimination and HCS classification results for the $1^{\text {st }}$ session data of the three subjects $\mathrm{D}, \mathrm{K}$, and $\mathrm{L}$ are shown in Fig. 3, 7, 11a) and b), respectively, while those for the $2^{\text {nd }}$ session data of the same three subjects are shown in Fig. 5, 9, 13a) and b), respectively. From the results shown in Fig. 3, 5, 7, 9, 11, and 13, it can be observed that the classification results based on the reduced number of features are also in good agreement with the actual trend of the HCS variations.
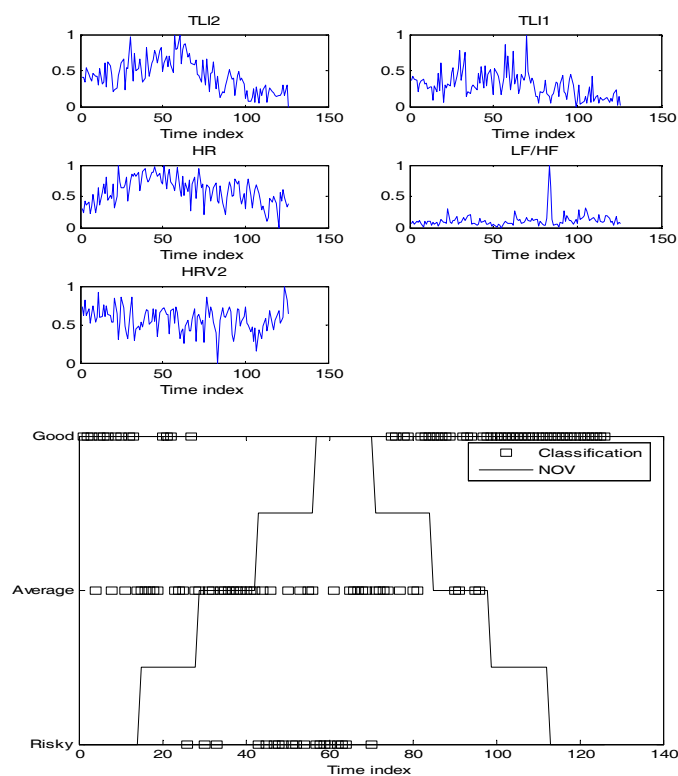

Fig. 2. The HCS features and classification results (subject D; s1): The temporal dynamics of the 5 features (upper) and HCS classification results (lower).
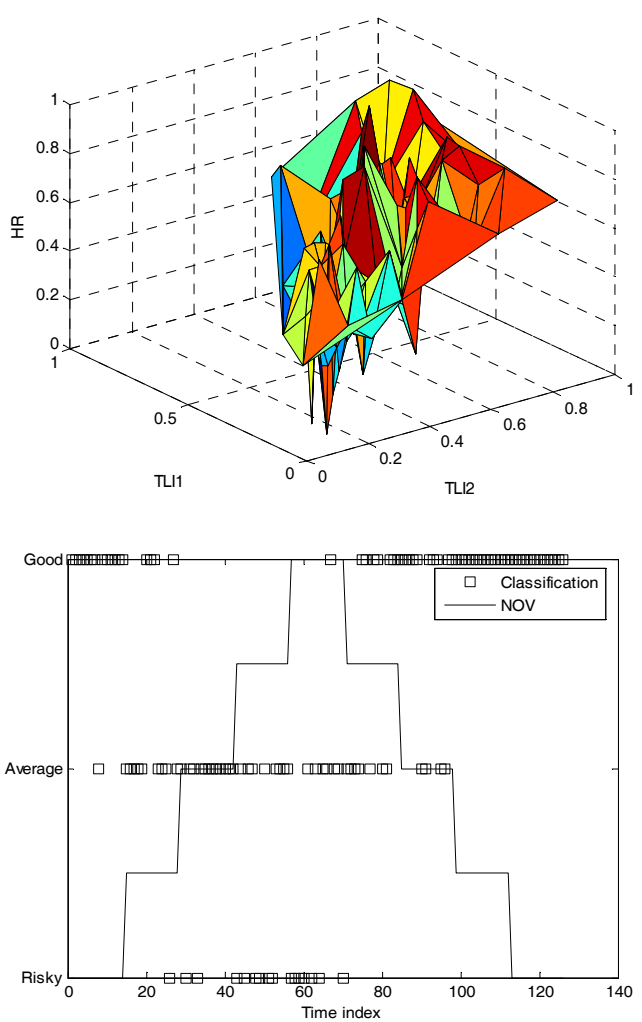

Fig. 3. The reduced 3D feature space (upper) and HCS classification results (lower): subject D; s1.
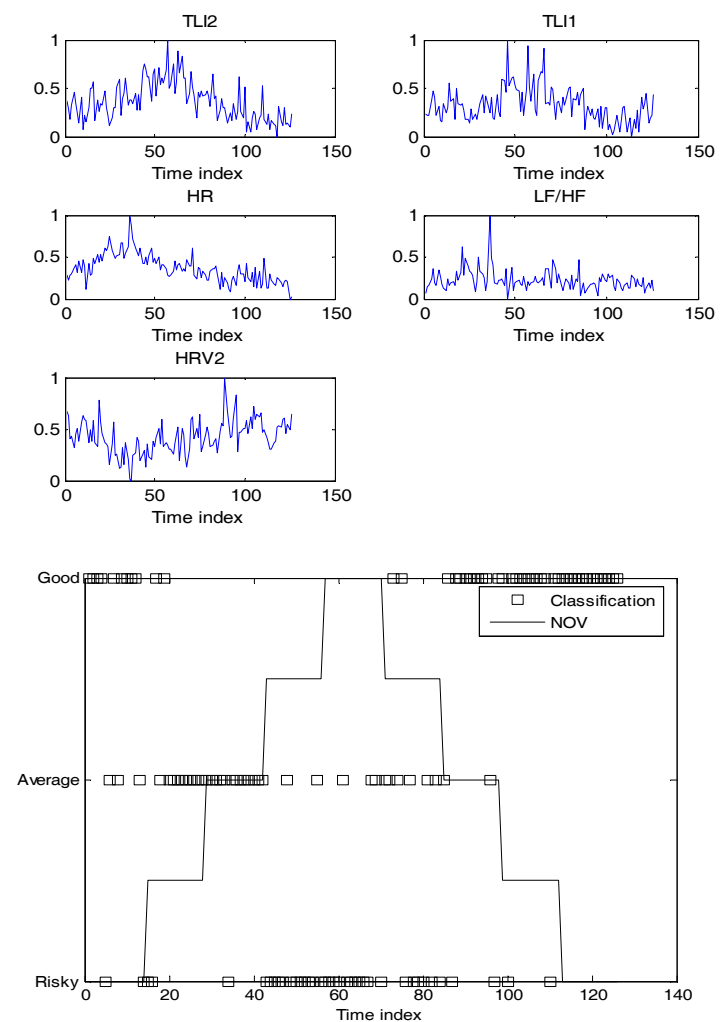

Fig. 4. The HCS features and classification results (subject D; s2): The temporal dynamics of the 5 features (upper) and HCS classification results (lower). 

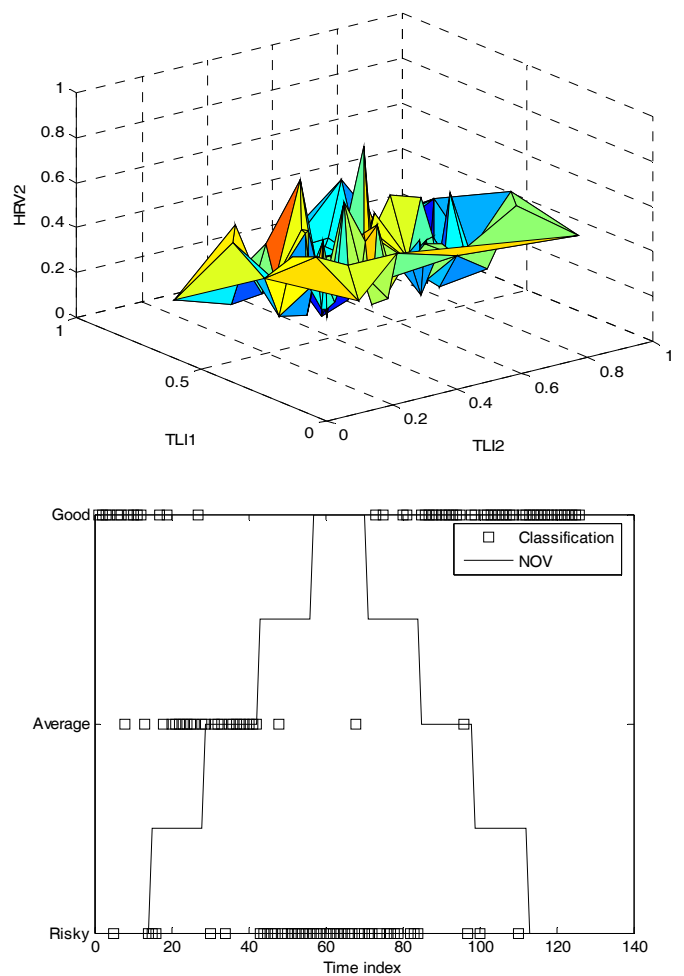

Fig. 5. The reduced 3D feature space (upper) and HCS classification results (lower): subject D; s2.

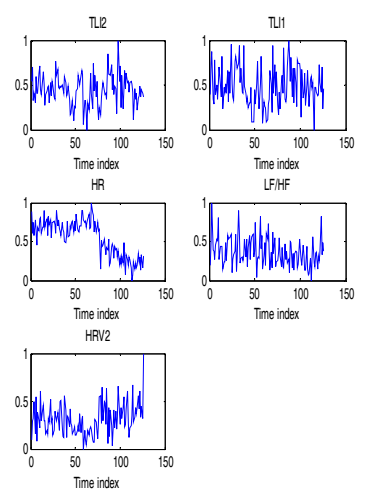

a)

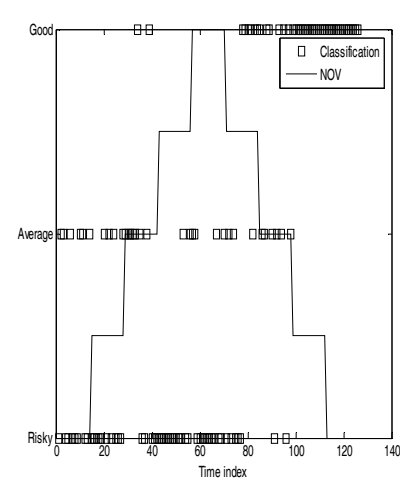

b)
Fig. 6. The HCS features and classification results (subject K; s1): a) The temporal dynamics of the 5 features; b) The HCS classification results.

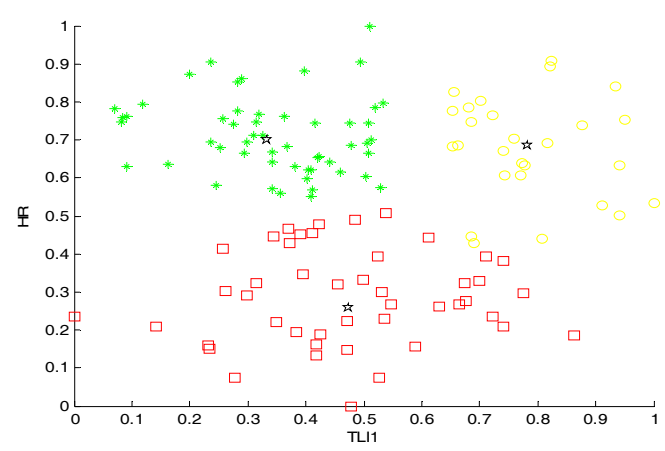

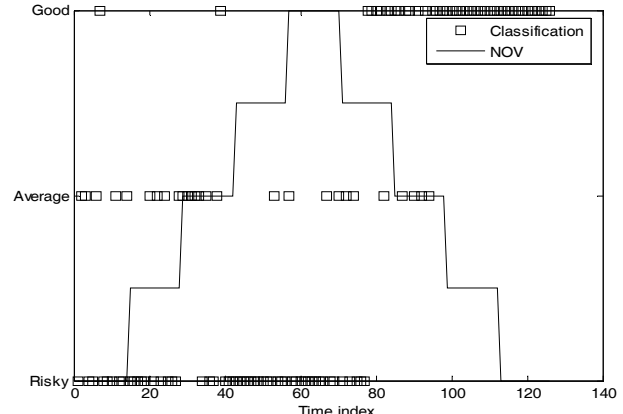

Fig. 7. The reduced 2D HCS feature plane (upper) and classification results (lower) for subject K; s1. Here the centers of three distinct OFS classes are indicated (upper).
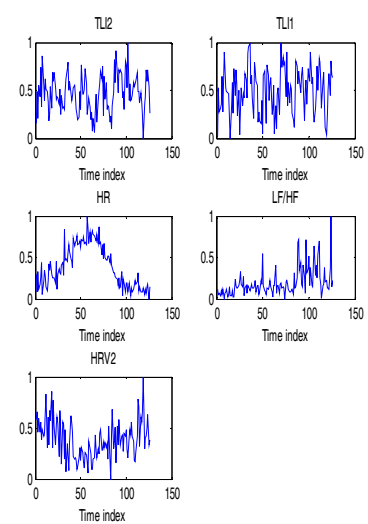

Time index

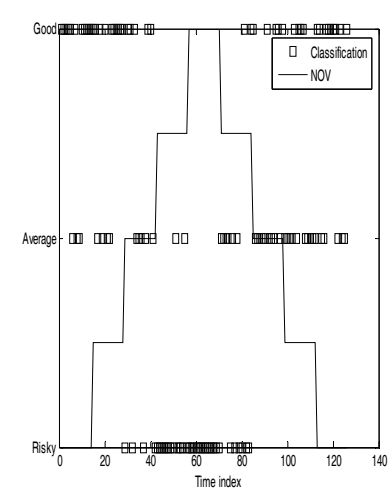

a)

b)

Fig. 8. The HCS features and classification results (subject K; s2): a) The temporal dynamics of the 5 features; b) The HCS classification results.

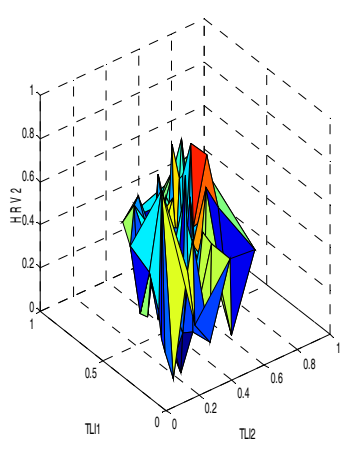

a)

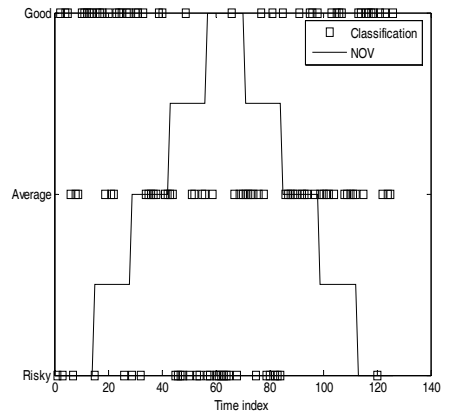

b)
Fig. 9. The reduced feature space and classification results (subject K; s2): a) The 3D HCS-feature space generated by data dimension-reduction (i.e., 2 features, LF/HF and HR, were eliminated); b) The HCS classification results. 

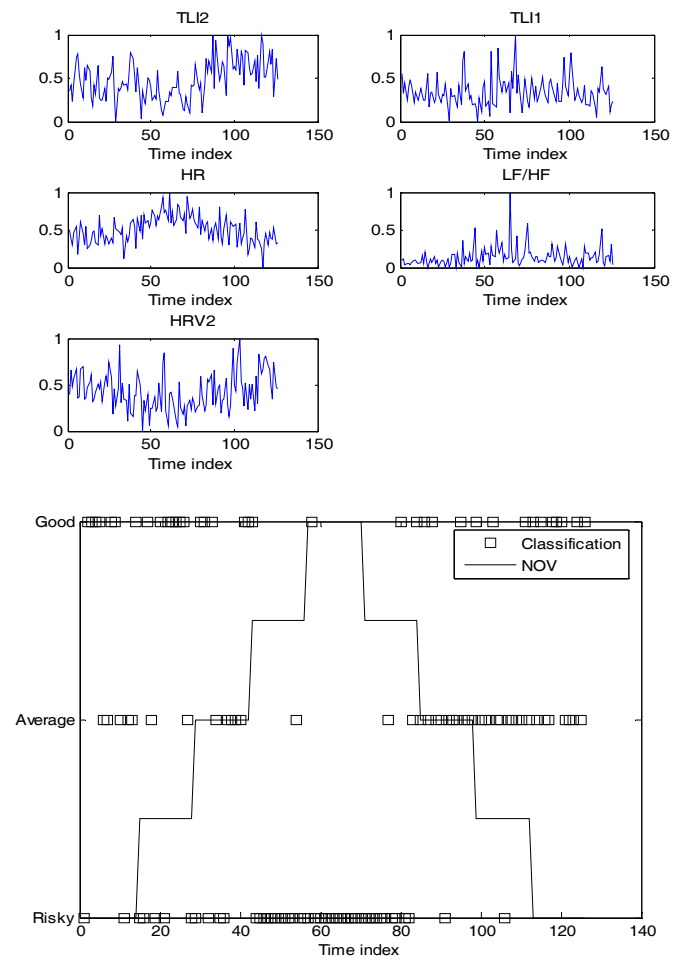

Fig. 10. The five candidate temporal features (upper) and HCS classification results (lower): subject L; s1.
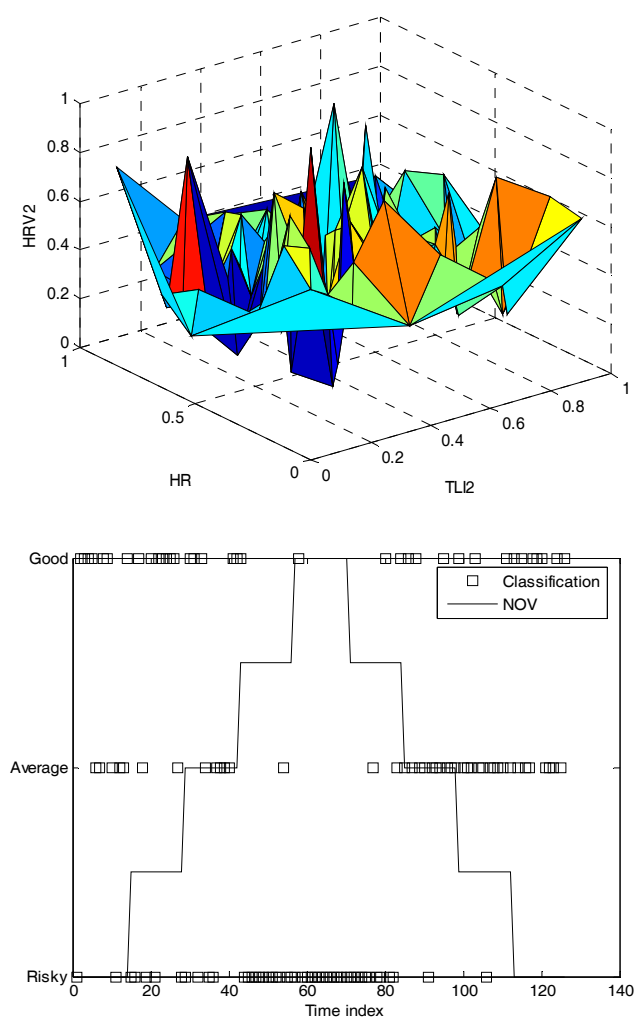

Fig. 11. The 3D feature space generated by dimensionality reduction (upper) and HCS classification results (lower): subject L; s1.

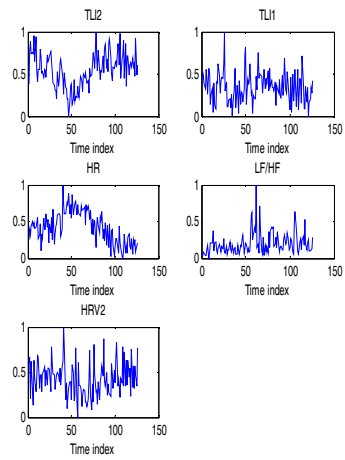

a)

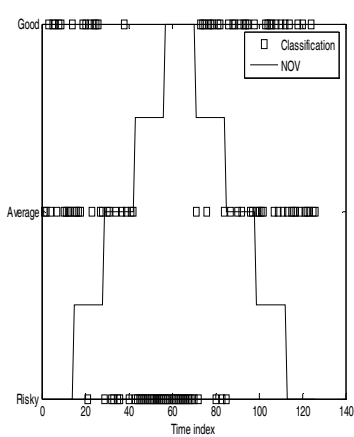

b)
Fig. 12. The HCS features and classification results (subject L; s2): a) temporal variation of 5 features; b) HCS classification results.

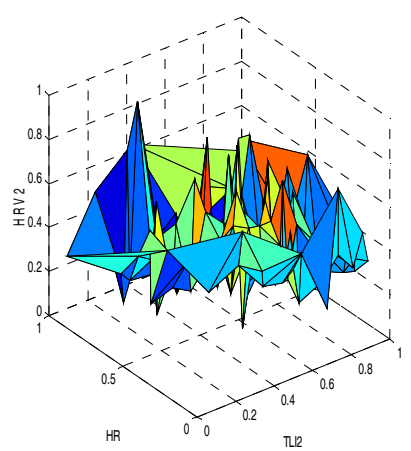

a)

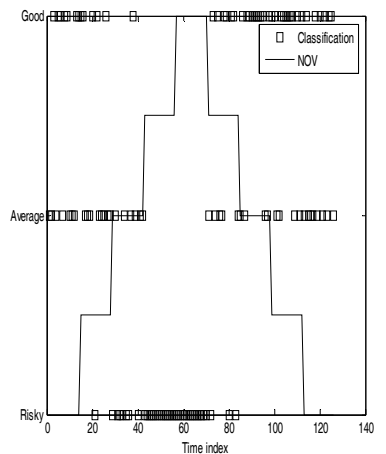

b)
Fig. 13. The reduced feature space and classification results (subject L; s2): a) The 3D HCS-feature space generated by data dimension-reduction (i.e., 2 features, $\mathrm{TLI}_{1}$ and $\mathrm{LF} / \mathrm{HF}$, were eliminated); b) The HCS classification results.

Furthermore, the subject-specific feature selection and the corresponding classification results of the both dataset for each subject before and after feature elimination are compared in Table 1, which gives not only the linear correlation coefficients ( $L C C$ ) between the classified HCS and the NOV parameter (see the $4^{\text {th }}$ and $5^{\text {th }}$ column), but also the classification consistency rate $(C C R)$ index before and after using feature selection (see the last column). In Table 1, the eliminated features are marked with "--", while the selected features marked by " $\sqrt{ }$ "; $s 1$ and s2 stand for the first and $2^{\text {nd }}$ dataset (measured in $1^{\text {st }}$ and $2^{\text {nd }}$ session of experiments), respectively. From the last row of Table 1, it is recognized that overall the most sensitive HCS feature is $\mathrm{TLI}_{2}$, followed by $\mathrm{HRV}_{2}, \mathrm{HR}$, and $\mathrm{TLI}_{1}$, while the index of $\mathrm{LF} / \mathrm{HF}$ was shown to be relatively insensitive to the variations in the HCS. It is also noted that the importance of each feature seems fairly different across subjects. From the LCC values shown in Table I, an obvious linear correlation was observed between the classified HCS and the actual change of the task-load in particular for subjects C, D, J, K and $\mathrm{L}$, which indicated that our classification results agree 
well with the actual HCS variations with the change of task load. From the CCR values given in the last row of Table I, among 126 data points the class labels of 122 pair of data (with a percentage of $96.83 \%$ ) are consistent for the $1^{\text {st }}$ session of subject L, while 111 pairs of data (with a percentage of $88.10 \%$ ) for the $2^{\text {nd }}$ session of subject D. It is also seen that the inconsistency of the data clustering results prior to and after the feature selection are small, and hence the validity of the proposed feature elimination (selection) approach is demonstrated.

Table 1. Comparison of HCS classification results with and without feature selection: 10 subjects with each having 2 measured datasets.

\begin{tabular}{|c|c|c|c|c|c|c|c|c|c|}
\hline \multirow{2}{*}{$\begin{array}{c}\text { Subject } \\
\text { code }\end{array}$} & \multirow{2}{*}{$\begin{array}{c}\text { Data } \\
\text { session } \\
\#\end{array}$} & \multicolumn{5}{|c|}{ Candidate physiological features } & \multirow{2}{*}{$\begin{array}{c}L C C \\
\text { without } \\
\text { feature } \\
\text { selection }\end{array}$} & \multirow{2}{*}{$\begin{array}{c}L C C \text { with } \\
\text { feature } \\
\text { selection }\end{array}$} & \multirow{2}{*}{$C C R$} \\
\hline & & $\mathrm{TLI}_{2}$ & $\mathrm{TLI}_{1}$ & HR & $\mathrm{LF} / \mathrm{HF}$ & $\mathrm{HRV}_{2}$ & & & \\
\hline \multirow{2}{*}{ A } & s1 & $\sqrt{ }$ & $\sqrt{ }$ & $\sqrt{ }$ & -- & -- & -0.1265 & -0.2481 & $70.63 \%$ \\
\hline & $\mathrm{s} 2$ & $\sqrt{ }$ & -- & $\sqrt{ }$ & -- & $\sqrt{ }$ & -0.2713 & -0.2345 & $88.10 \%$ \\
\hline \multirow{2}{*}{$\mathrm{C}$} & s1 & $\sqrt{ }$ & -- & $\sqrt{ }$ & -- & -- & -0.5437 & -0.4438 & $84.13 \%$ \\
\hline & $\mathrm{s} 2$ & $\sqrt{ }$ & $\sqrt{ }$ & -- & -- & $\sqrt{ }$ & -0.5255 & -0.5418 & $78.57 \%$ \\
\hline \multirow{2}{*}{$\mathrm{D}$} & s1 & $\sqrt{ }$ & $\sqrt{ }$ & $\sqrt{ }$ & -- & -- & -0.6131 & -0.6185 & $94.44 \%$ \\
\hline & $\mathrm{s} 2$ & $\sqrt{ }$ & -- & $\sqrt{ }$ & -- & $\sqrt{ }$ & -0.6396 & -0.6652 & $88.10 \%$ \\
\hline \multirow{2}{*}{$\mathrm{E}$} & $\mathrm{s} 1$ & -- & $\sqrt{ }$ & -- & $\sqrt{ }$ & $\sqrt{ }$ & -0.0108 & -0.0483 & $77.78 \%$ \\
\hline & $\mathrm{s} 2$ & - & $\sqrt{ }$ & $\sqrt{ }$ & - & $\sqrt{ }$ & -0.0777 & -0.0026 & $90.48 \%$ \\
\hline \multirow{2}{*}{$\mathrm{F}$} & s1 & $\sqrt{ }$ & -- & -- & -- & $\sqrt{ }$ & -0.1078 & -0.2471 & $65.87 \%$ \\
\hline & $\mathrm{s} 2$ & $\sqrt{ }$ & $\sqrt{ }$ & -- & -- & $\sqrt{ }$ & -0.2046 & -0.0824 & $92.86 \%$ \\
\hline \multirow{2}{*}{$\mathrm{G}$} & s1 & $\sqrt{ }$ & $\sqrt{ }$ & $\sqrt{ }$ & -- & -- & -0.1315 & -0.1179 & $88.10 \%$ \\
\hline & $\mathrm{s} 2$ & $\sqrt{ }$ & -- & $\sqrt{ }$ & -- & $\sqrt{ }$ & -0.3899 & -0.2723 & $76.19 \%$ \\
\hline \multirow{2}{*}{$\mathrm{H}$} & s1 & $\sqrt{ }$ & $\sqrt{ }$ & -- & -- & $\sqrt{ }$ & -0.4899 & -0.4814 & $88.89 \%$ \\
\hline & s2 & $\sqrt{ }$ & -- & $\sqrt{ }$ & -- & $\sqrt{ }$ & -0.527 & -0.4002 & $77.78 \%$ \\
\hline \multirow{2}{*}{$\mathrm{J}$} & s1 & -- & $\sqrt{ }$ & $\sqrt{ }$ & -- & $\sqrt{ }$ & -0.416 & -0.3869 & $91.27 \%$ \\
\hline & $\mathrm{s} 2$ & $\sqrt{ }$ & $\sqrt{ }$ & -- & -- & $\sqrt{ }$ & -0.6008 & -0.5083 & $89.68 \%$ \\
\hline \multirow{2}{*}{ K } & s1 & -- & $\sqrt{ }$ & $\sqrt{ }$ & -- & -- & -0.3455 & -0.3655 & $92.86 \%$ \\
\hline & $\mathrm{s} 2$ & $\sqrt{ }$ & $\sqrt{ }$ & -- & -- & $\sqrt{ }$ & -0.7202 & -0.4768 & $82.54 \%$ \\
\hline \multirow{2}{*}{ L } & s1 & $\sqrt{ }$ & -- & $\sqrt{ }$ & -- & $\sqrt{ }$ & -0.5484 & -0.5008 & $96.83 \%$ \\
\hline & s2 & $\sqrt{ }$ & -- & $\sqrt{ }$ & -- & $\sqrt{ }$ & -0.5244 & -0.5752 & $86.51 \%$ \\
\hline \multicolumn{2}{|c|}{ \# of selection } & $16 / 20$ & $12 / 20$ & $13 / 20$ & $1 / 20$ & $15 / 20$ & & & \\
\hline
\end{tabular}

\section{CONCLUSIONS}

Based on a series of the measured physiological data, 5 key features which are sensitive to the HCS variations are calculated in this paper. Then the FCM algorithm is adopted to realize the fuzzy classification of the momentary HCS change under variable mental workload conditions. The cyclical loading design scheme suggested also allows us to evaluate the HCS classification performance without the explicit knowledge of the target classes. The results for 10 subjects have shown that the hybrid-data-based fuzzy pattern classification technique can recognize individually the real variations in the HCS. The correlation analysis showed that the classified HCS clearly reflects the gradual change of the HCS in response to the stepwise variations of the task-load 
(i.e., the level of manual control task difficulty) specified by the cyclical loading scheme. The feature selection (or called data dimensionality-reduction) was also performed based on a criterion of the cluster centers generated by the FCM algorithm. Our results have also shown a significant individual difference on the subject-specific HCS-related patterns finally selected. Moreover, the unimportant feature elimination procedure ensures comparable classification accuracy with enhanced computational efficiency, which may be desirable for online real-time HCS classification.

Due to the complex and at most partially known relationship between the HCS and the psychophysiological responses of the human operator, the entirely data-based method has to be used for its investigation. Although the fuzzy logic based paradigm was shown to be effective for the HCS classification problem by this work, some further work needs to be done in the following two directions: 1) The improved classification algorithm: For instance, two possible problems may arise: i) How to extract the individually optimal or near-optimal HCS features for each subject? ii) How to improve the computing efficiency aspect of the FCM algorithm, utilized in this paper, to make it more tailored to real-time online classification of the HCS required by the practical situations? 2) The measured data: To further improve the generalization (or predictive) accuracy and reliability of the classification algorithms, more sessions of experimental data for each subject should be available. On the other hand, the data measured from more subjects are required to study the individual difference between subjects and the possibility of designing a generic HCS classifier for certain group of subjects with similar physical characteristics.

\section{ACKNOWLEDGMENTS}

This work was supported by National Natural Science Foundation of China under Grant No. 61075070 and Key Grant No. 11232005.

\section{REFERENCES}

Bezdek, J.C. (1981), Pattern Recognition with Fuzzy Objective Function Algorithms, New York: Plenum.

Hockey, G.R.J. (2003), Operator functional state: The assessment and prediction of human performance degradation in complex tasks, IOS Press.

Hockey, G.R.J., Wastell, D.G., and Sauer J. (1998), Effects of sleep deprivation and user-interface on complex performance: A multilevel analysis of compensatory control, Human Factors, Vol. 40, pp. 233-253.

Jasper, H.H. (1958), Report of the committee on methods of clinical examination in electroencephalography, Electroencephalography and Clinical Neurophysiology, Vol. 10, pp. 370-375.

Nickel, P., Roberts, A.C., and Hockey G.R.J. (2005), Assessment of high risk operator functional state markers in dynamical systems-preliminary results and implications, in Proc. of Human Factors and Ergonomics Society Europe Chapter Annual Meeting, Turin, Italy, Oct. 26-28.

Nickel, P., Hockey, G.R.J. Roberts, A.C., and Roberts M.H. (2006), Markers of high risk operator functional state in adaptive control of process automation, in Proc. of IEA.

Wilson, G.F. (1999), Operator functional state classification using neural networks with combined psychological and performance measures, in Proc of the $43^{\text {rd }}$ HFES Annual Meeting, pp. 1099-1102.

Zhang, Jian-Hua, Wang, Xing-Yu, Mahfouf M., and Linkens D.A. (2008), Use of heart rate variability analysis for quantitatively assessing operator's mental workload, in Proc. of Int. Conf. Biomedical Engineering \& Informatics (BMEI), Vol. 1, pp. 668-672, Sanya, China. 